\title{
THE TEMPORAL AND SPECTRAL CHARACTERISTICS OF "FAST RISE AND EXPONENTIAL DECAY" GAMMA-RAY BURST PULSES
}

\author{
Z. Y. Peng ${ }^{1}$, Y. Yin ${ }^{2}$, X. W. Bi $^{3}$, X. H. Zhao ${ }^{4}$, L. M. Fang ${ }^{5}$, Y. Y. Bao ${ }^{6}$, L. Ma ${ }^{1, *}$
}

Received — ; accepted

\footnotetext{
${ }^{1}$ Department of Physics, Yunnan Normal University, Kunming 650092, China; pzy@ynao.ac.cn

*Corresponding author, astromali@126.com

${ }^{2}$ Department of Physics, Liupanshui Normal College, Liupanshui 553004, China

${ }^{3}$ Department of Physics, Honghe College, Mengzi, 661100, China

${ }^{4}$ National Astronomical Observatories/Yunnan Observatory, Chinese Academy of Sciences, P. O. Box 110, Kunming 650011, China

${ }^{5}$ Department of Physics, Guangdong Institute of Education, Guangzhou 510303, China

${ }^{6}$ Department of Physics, Yuxi Normal College, Yuxi 653100, China
} 


\begin{abstract}
In this paper we have analyzed the temporal and spectral behavior of 52 Fast Rise and Exponential Decay (FRED) pulses in 48 long-duration gamma-ray bursts (GRBs) observed by the CGRO/BATSE, using a pulse model with two shape parameters and the Band model with three shape parameters, respectively. It is found that these FRED pulses are distinguished both temporally and spectrally from those in long-lag pulses. Different from these long-lag pulses only one parameter pair indicates an evident correlation among the five parameters, which suggests that at least $\sim 4$ parameters are needed to model burst temporal and spectral behavior. In addition, our studies reveal that these FRED pulses have correlated properties: (i) long-duration pulses have harder spectra and are less luminous than short-duration pulses; (ii) the more asymmetric the pulses are the steeper the evolutionary curves of the peak energy $\left(E_{p}\right)$ in the $\nu f_{\nu}$ spectrum within pulse decay phase are. Our statistical results give some constrains on the current GRB models.
\end{abstract}

Subject headings: gamma rays: bursts - method: statistical 


\section{INTRODUCTION}

The temporal profiles of Gamma-ray burst (GRB) are very diverse in morphology but the spectra could be fitted with a single simple Band model (Band et al. 1993). The spectral parameters (the power-law indices and the peak energy in the $\nu f_{\nu}$ spectrum) are then used to infer the GRB emission and particle acceleration mechanisms. However, the signatures of the gamma-ray epoch of the burst are hidden in the time evolution of the light curve and in its spectral behavior. The individual emission episodes (pulses) that complex light curves are believed to consist of reflect the behavior of central engine. Due to the overlapping of pulses in most bursts, especially that bright ones, only a small fraction of all bursts consist of long, smooth and well-shaped pulse, often with a fast rise and a exponential decay (FRED), while others exhibit very complex and jagged light curves. Therefore accurate study of individual pulse behavior is often difficult. However, some dimmer bursts with lower signal-to-noise ratios usually have simpler temporal structure may be easy to model (Norris et al. 2005, hereafter Paper I). The investigations of these pulses are useful, which might lead to a deeper understanding of the creation of the gamma-rays by giving clues to and constraining physical models.

Many authors have studied the temporal and spectral properties of long-duration $\left(T_{90}>2 \mathrm{~s}\right)$ GRB pulses, a number of characteristics of these pulses are revealed. The impressive results include: e.g. (1) the temporal asymmetry of pulses in GRBs, that is longer decay than rise rates, (2) hard-to-soft spectral evolution, and (3) energy dependence of the pulse duration, broadening at lower energies (e.g., Norris et al. 1996; Ryde 2005; Golenetskii et al. 1983; Borgonovo \& Ryde 2001; Kouveliotou et al. 1993).

However, most of these studies focus on the pulses in bright bursts. Stern et al. (1999) investigated a complexity-brightness correlation in GRB and found that the average profiles

of dim bursts were less complex than that of bright bursts. Based on this Paper I analyzed 
the temporal and spectral behavior of some long-lag bursts, which tend to be dim but also to have relatively simple temporal structures. They found that pulses in long-lag bursts are distinguished both temporally and spectrally from those in bright bursts: (1) the pulses in long-lag bursts are few in number, $(2)$ the durations are $\sim 100$ times wider (tens of seconds) than those of bright bursts, (3) the peak energy $E_{p}$ in $\nu f(\nu)$ is lower, and (4) the long-lag bursts have harder low-energy spectra and softer high-energy spectra.

Kocevski et al. (2003) analyzed the time profiles of 76 FRED pulses with the peak flux greater than that long-lag pulses. They only considered the temporal behavior of these pulses and did not analyze the spectral properties. Employing this sample Peng et al. (2009a) (hereafter Paper II) studied the spectral behavior of these FRED pulses that are bright enough to perform spectral analysis. They focused their attentions on the

evolutionary slope of peak energy $E_{p}$ within the pulse decay phase and found that the slope is correlated with several spectral parameters.

In the present work, we would like to employ the sample presented by Paper II to investigate the temporal and spectral properties of these FRED pulses. If these bursts are distinguished from long-lag bursts temporally and spectrally is our another motivation. In Section 2, we present the sample description. The temporal and spectral profile analysis are given in Section 3. Discussion and conclusions are presented in the last section.

\section{SAMPLE DESCRIPTION}

Paper II used two samples provided by Kocevski et al. (2003) and Norris et al. (1999) to investigate the evolutionary slope of $E_{p}$ in FRED pulses. The main selected criterions of the two samples of Paper II are: (1) the data are provided by the BATSE instruments on board the CGRO spacecraft and the duration is greater than $2 \mathrm{~s}\left(T_{90}>2 \mathrm{~s}\right)$; (2) exhibited 
clean, single-peaked events or, in the case of multi-peaked bursts, pulses that were well distinguished and separable from each other; (3) the peak flux is greater than 1.8 photon $\mathrm{cm}^{-2} \mathrm{~s}^{-1}$ on a $256 \mathrm{~ms}$ timescale. The time-resolved and time-integrated spectra of the two samples were fitted with the Band and Compton model, respectively. Based on these fitting parameters Paper II studied the evolutionary slope of $E_{p}$ as well as the correlations between the slope and the spectral parameters. Their analysis showed the two samples share approximately the same statistical properties, which can be found from Figure 1 to Figure 9 in Paper II (for more details of the samples and the spectral modeling, one can refer to Kocevski et al. 2003, Norris et al. 1999, and Paper II). Therefore, we only select, in this paper, the sample fitted by Band model to investigate the temporal and spectral characteristics of FRED pulses, which includes 56 single pulses.

\section{TEMPORAL PROFILE AND TIME-INTEGRATED SPECTRA ANALYSIS}

\subsection{Temporal Profile Analysis}

Once these pulses have been selected we use the pulse model of Paper I to fit them. The pulse model can be rewritten as follows:

$$
I(t)=A \lambda \exp \left[-\tau_{1} /\left(t-t_{s}\right)-\left(t-t_{s}\right) / \tau_{2}\right]
$$

where $t$ is time since trigger, $A$ is the pulse amplitude, $t_{s}$ is the pulse start time, $\tau_{1}$ and $\tau_{2}$ are characteristics of the pulse rise and pulse decay, and $\lambda=\exp \left[2\left(\tau_{1} / \tau_{2}\right)\right]^{1 / 2}$.

Similar to Peng et al. (2006, 2009b) and Hakkila et al. (2008) we also use nonlinear least squares routine MPFIT to fit these pulses. It is based on the well-known and tested MINPACK-1 FORTRAN package of routines available at www.netlib.org. Moreover, MPFIT functions may permit you to fix any function parameters, as well as to set simple 
upper and lower parameter bounds. To obtain an intuitive view of the result of the fit, we develop and apply an interactive IDL routine for fitting pulses in bursts, which allows the user to set and adjust the initial pulse parameters manually before allowing the fitting routine to converge on the best-fitting model via the reduced $\chi^{2}$ minimization. The background-subtracted light curves combined four channels are fitted with the pulse model. The fits are examined many times to ensure that they are indeed the best ones. The fitting $\chi^{2}$ per degree of freedom larger than 2.5 are rejected. In the end there are 52 pulses are included in our sample.

We demonstrate two fit results with the largest values of $\chi^{2}$ (GRB 980301 (BATSE trigger 6621)) and with smallest value (GRB 931128 (BATSE trigger 2665)) in Figure 1. The distributions of $\chi^{2}$ per degree of freedom for our sample are displayed in Figure 2. The narrow distribution of the $\chi^{2}$ values indicates that two-exponential model is sufficient to model the pulse light curves.

According to the fitted parameters we can obtain the two shape parameters, width $w$ and asymmetry $k$. Following Paper I we find the pulse width measured between the two $1 / e$ intensity points, $w=\triangle \tau_{1 / e}=\tau_{2}(1+2 \ln \lambda)^{1 / 2}$. The form of the pulse asymmetry $k=\tau_{2} / w$. Quilligan et al. (2002) found that the full-widths at half-maximum (FWHM) of GRB pulse is log-normal distribution. It is found the distribution of $w$ is also log-normal (see Figure 3) but the distribution of $k$ is normal (Figure 3). The parameters of the best log-normal and normal fits are given in Table 1.

In addition, we find no significant correlation between the width and asymmetry, which is consistent with the result of long-lag pulses. The widths of these FRED pulses are distinguished from the long-lag pulses since the analysis of long-lag pulse performed by Paper I showed the average width is larger than $10 \mathrm{~s}$. Whereas the difference of the mean value of the asymmetry between the FRED pulses and the long-lag pulses is not evident 

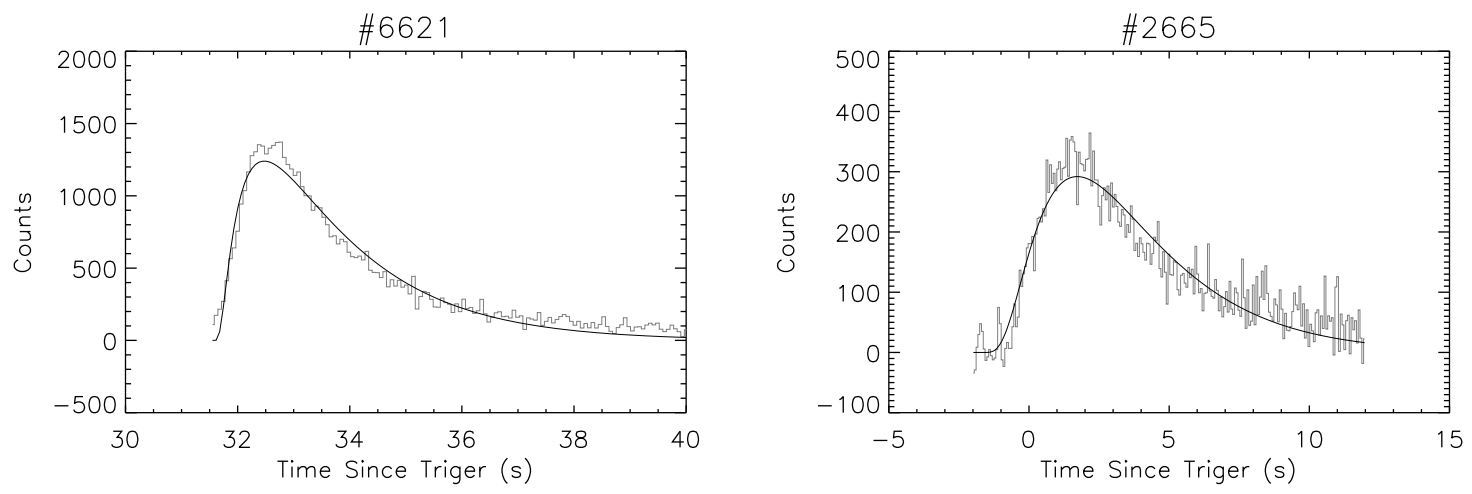

Fig. 1. - The plots of the fitting result of two pulse with the largest value of $\chi^{2}$ (left panel) and with the smallest value of $\chi^{2}$ (right panel) in our sample.

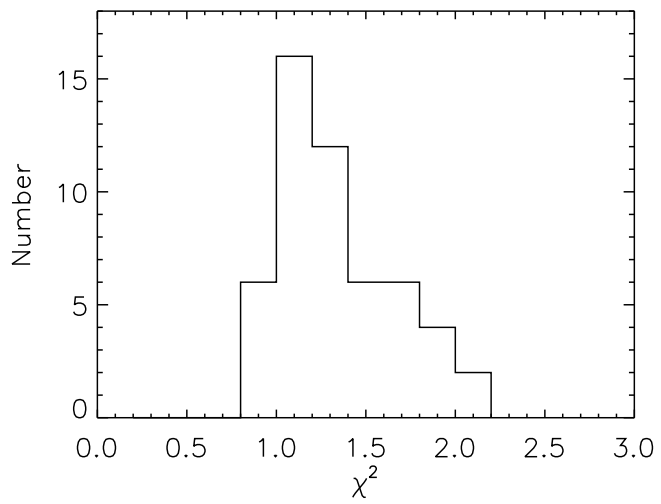

Fig. 2.- Histograms for the distribution of $\chi^{2}$ in our sample. 
(see, Table 1). Actually considering the standard deviations $(\sigma)$ and the number of pulses in each sample (\#52 FREDs and \#35 long-lag from Paper I) the mean k's are within 1 standard error of the sample mean. In other words, they are equal within uncertainties.

\subsection{Spectral Profile Analysis}

Paper II described in detail the spectral modeling for the single pulses and analyzed the evolutionary slope, $S$, during the decay phase of the FRED pulses. In addition, they

examined the relations between spectral parameters and $S$. In this section, let us first check the distributions of time-integrated spectral parameters for our sample. Figure 4 indicates the distributions of the spectral parameters, $E_{p}, \alpha, \beta$. The corresponding mean value and standard deviation are listed in Table 1. Note that the spectral parameters come from spectra integrated over a FRED pulse rather than an entire burst.

Previous study showed the distribution of $E_{p}$ integrated over a burst is best described by a log-normal distribution (Quilligan et al. 2002). We find $E_{p}$ distribution for the single pulses is also log-normal. Compared with these long-lag pulse the $E_{p}$ of FRED pulse is much greater (see Table 1). While for the low-energy index the distribution is approximately normal and the spectra of most FRED pulses are much steeper than those of the long-lag pulses. Whereas for the high-energy index the spectra of most of FRED pulses are a little flatter than those of the long-lag pulses (see Table 1).

\subsection{The Relation Between The Temporal And Spectral Parameters}

Paper I have examined the relations between the temporal and spectral parameters and showed: (1) no clear correlation between the low-energy index $\alpha$ and the width of pulse $w$ is indicated; (2) there is a suggestion that $\alpha$ is correlated with pulse asymmetry; (3) the 

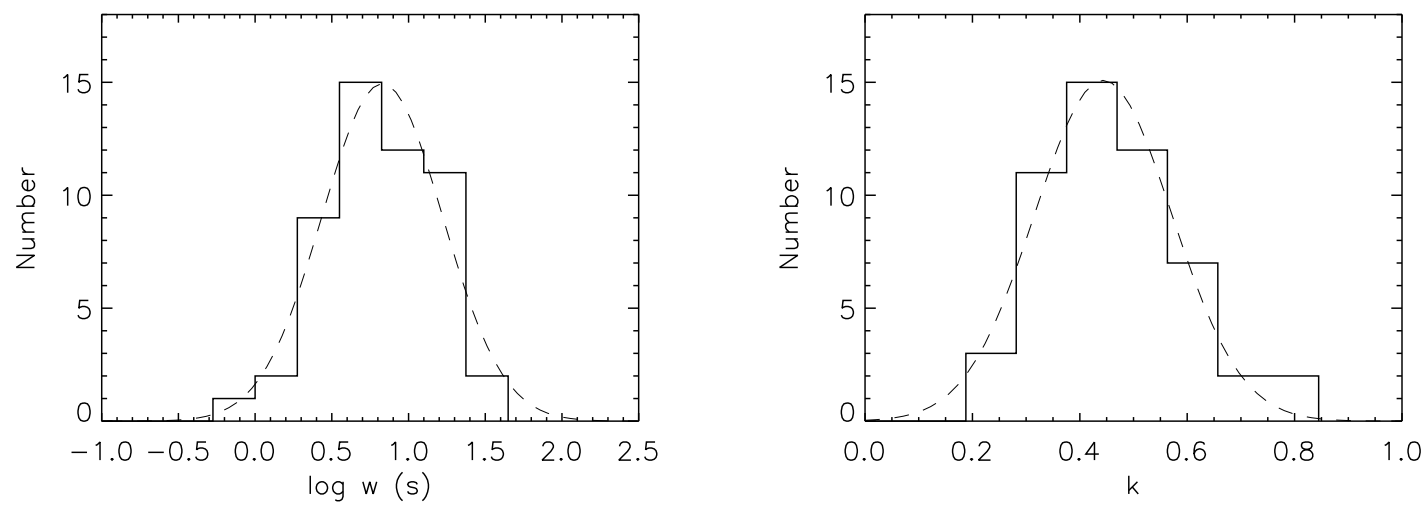

Fig. 3.- Distributions of the pulse width, $w$ (left panel) and pulse asymmetry, $k$ (right panel) in our sample, where the curves represent the Gaussian fit to the two distributions.

Table 1. A comparison of temporal and spectral parameters for the FRED and long-lag pulses.

\begin{tabular}{cccccc}
\hline \hline & \multicolumn{2}{c}{ FRED pulse } & & \multicolumn{2}{c}{ Long-lag pulse $^{a}$} \\
\cline { 2 - 3 } \cline { 5 - 6 } property & $\mu$ & median & & $\mu$ & median $^{*}$ \\
\cline { 2 - 3 }$w(\mathrm{~s})$ & $6.74 \pm 2.47$ & 6.45 & & $15.18 \pm 13.75$ & 12.96 \\
$k$ & $0.44 \pm 0.13$ & 0.46 & & $0.40 \pm 0.19$ & 0.41 \\
$E_{p}(\mathrm{keV})$ & $158.49 \pm 79.67$ & 161.40 & & $109.89 \pm 64.50$ & 110.75 \\
$\alpha$ & $-0.89 \pm 0.52$ & -0.87 & & $-0.46 \pm 0.65$ & -0.47 \\
$\beta$ & $-2.60 \pm 0.37$ & -2.63 & & $-2.74 \pm 0.25$ & -2.80 \\
\hline
\end{tabular}

Note. $-{ }^{a}$ Reference for the long-lag pulse data: Norris et al. (2005), i.e., Paper I. 

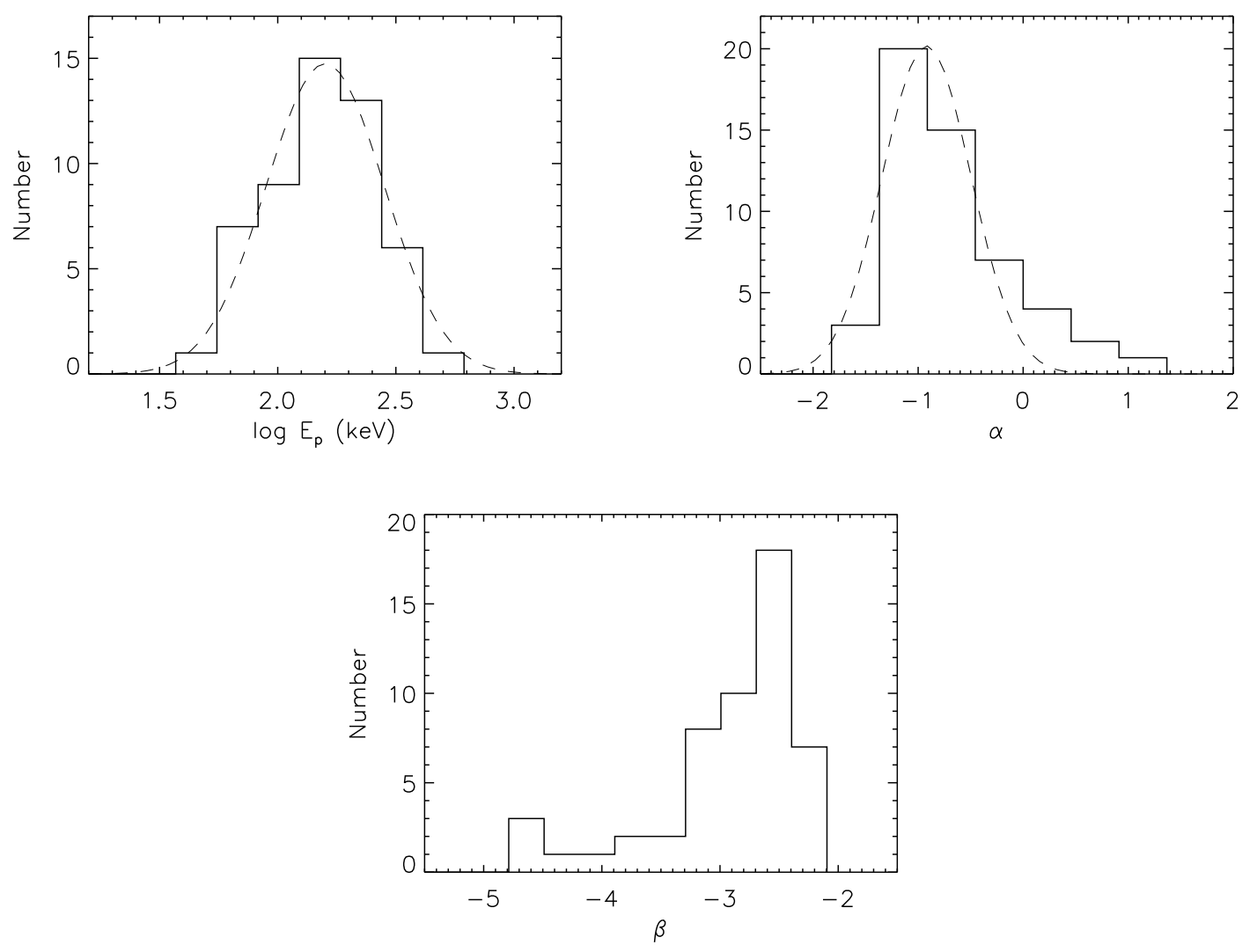

Fig. 4.- Distributions of the pulse peak energy, $E_{p}$, low-energy index, $\alpha$, and high-energy index, $\beta$ in our sample, where the curves represent the Gaussian fit to the two distributions. 
$E_{p}$ appears to be uncorrelated with any temporal parameters; (4) neither of two temporal parameters is correlated with high-energy index $\beta$. We re-examine the relations and find that the results are also established for our sample except that two differences from that of long-lag pulse (Paper I). The first difference is that the relation between $\alpha$ and $w$. A correlated relation between them is suggested for our sample (see, Figure 5 and Table 2). The second difference that there seems no correlation between $\alpha$ and $k$ (see Figure 5 and Table 2). The other parameter pairs, $E_{p}$ versus $\alpha, E_{p}$ versus $\beta, E_{p}$ versus $w, E_{p}$ versus $k, \alpha$ versus $\beta, w$ versus $\beta$, and $k$ versus $\beta$, are not correlated with each other.

Paper II have studied the relations between the decay slope of pulse, $S$, and the spectral parameters. In this work we mainly check the relations between $S$ and two temporal parameters as well as photon flux and temporal parameters.

Figure 6 (left panel) shows the relation between the $S$ and $w$. A anti-correlation between them is identified for our samples (also see Table 2). In addition, a correlation between the $S$ and pulse asymmetry $k$ is suggested in Figure 6 (right panel) and Table 2. Figure 7 shows a similar picture for the photon flux versus two temporal parameters. A clear correlated relation between photon flux and $w$ is identified, but there seems no evident correlation between the photon flux and $k$ (also see Table 2).

Table 2. Correlations of the six parameter pairs.

\begin{tabular}{ccc}
\hline \hline & & \\
Parameter Pair & $R_{S}$ & $P_{S}$ \\
\hline$w-\alpha$ & 0.49 & 0.0006 \\
$k-\alpha$ & -0.0018 & 0.99 \\
$w-S$ & -0.28 & 0.04 \\
$k-S$ & 0.49 & $<10^{-4}$ \\
$w-F$ & -0.53 & $<10^{-4}$ \\
$k-F$ & -0.17 & 0.22 \\
\hline
\end{tabular}



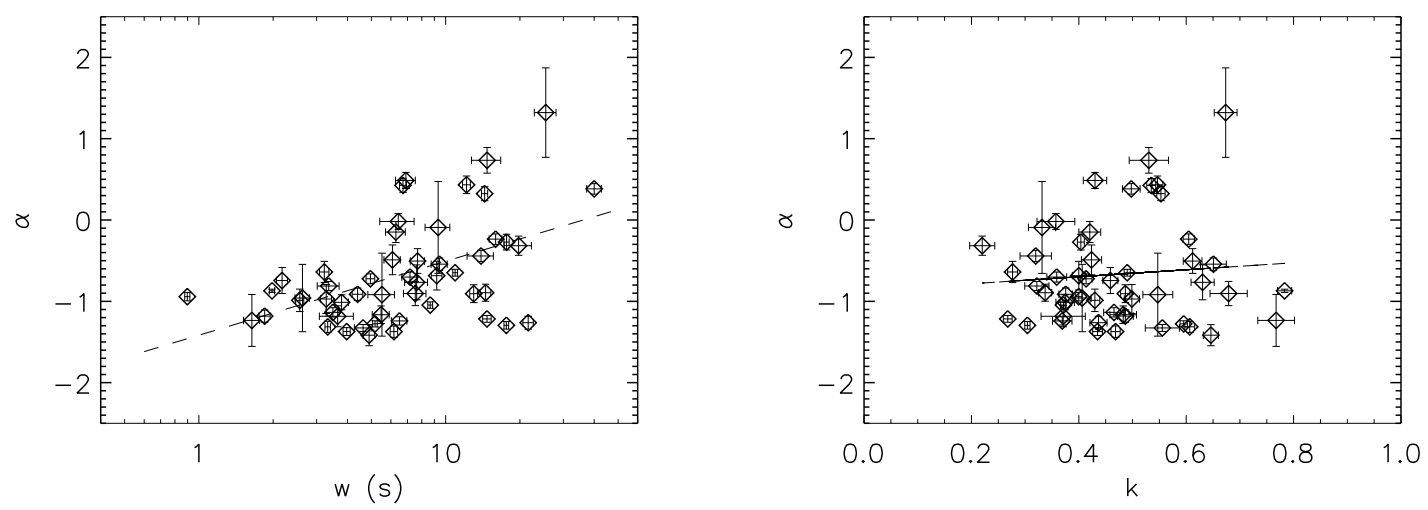

Fig. 5. - Spectral shape parameter $\alpha$ vs. pulse width $w$ (left panel) as well as $\alpha$ vs. pulse asymmetry $k$ (right panel), where the long dashed lines represent the best fitting lines.
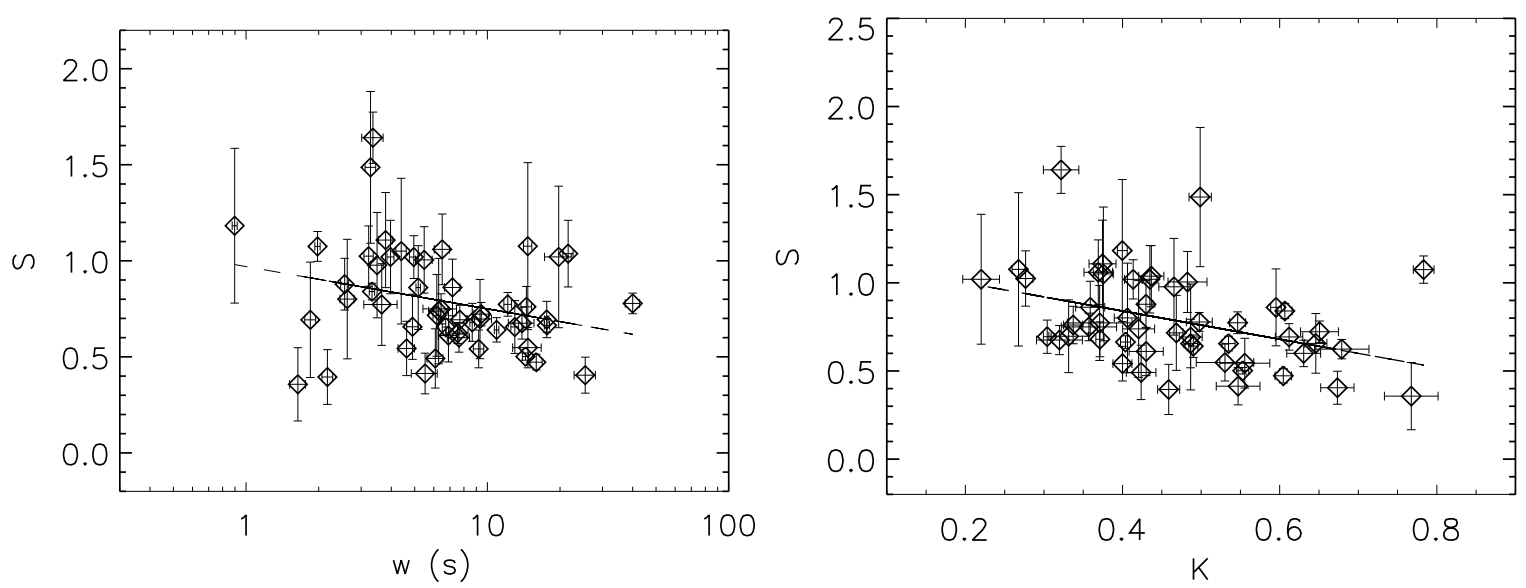

Fig. 6.- The $E_{p}$ evolutionary slope during the pulse decay phase, $S$, vs. pulse width $w$ (left panel) and pulse asymmetry $k$ (right panel), where the long dashed lines represent the best fitting lines. 
Based on the above analysis the five fundamental temporal and spectral shape parameters $w, k, \alpha, \beta$, and $E_{p}$ do not show compelling evidence for any pairwise correlation, except for a correlation between $w$ and $\alpha$. This implies that at least four independent physical parameters are required to determine pulse behavior in the energy band $\sim 25$ $2000 \mathrm{keV}$, which is different from that of long-lag pulse investigated by Paper I.

\section{Discussion and Conclusions}

By studying the temporal and spectral characteristics of FRED GRB pulses we first show that the FRED pulse is distinct from that long-lag pulse temporally and spectrally:

(1) the average width of FRED pulses $(8.75 \mathrm{~s})$ is below the corresponding values of long-lag (the average width is above $10 \mathrm{~s}$, Paper I); (2) the average peak energy, $E_{p}$, in the $\nu f_{\nu}$ is $158 \mathrm{keV}$ which is also greater than the long-lag pulse $(110 \mathrm{keV})$; (3) the low-energy indices obtained from FRED pulses are softer than that of long-lag pulses; (4) while the high-energy indices of FRED pulses are slightly harder than that of long-lag pulses. Therefore, these long-lag, wide-pulse GRBs and these general sample of GRB pulses may represent different sub-class with generally different physical properties. But the difference of the pulse asymmetry between the FRED and long-lag pulses is not significant.

Analysis of relations of temporal and spectral shape parameters suggests that they have no visible correlation except that the pulse width is correlated with lower-energy index $\alpha$, which indicates that at least four parameters are needed to model burst temporal and spectral behavior. The inconsistency of correlations between $w$ and $\alpha$ as well as pulse asymmetry $k$ and $\alpha$ with that of the long-lag burst studied by Paper I may be caused by the sample size. Our sample consisting of 52 pulses is a factor of 2 larger than that of long-lag bursts. Ryde et al. (2005) and Ryde (2005) showed a similar relation that hard spectra (with large spectral power-law indices $\alpha$ ) give the largest lags. Moreover, Paper I pointed 
out that pulse width is strongly correlated with spectral lag and these two parameters may be viewed as mutual surrogates. If this is the case we tend to believe that there are indeed correlation between $w$ and $\alpha$. Our analysis confirms that $k$ is not correlated with $\alpha$. Another possibility is that the characteristic of the FRED pulse is indeed different from long-lag pulse as shown above.

The anti-correlation between $w$ and photon flux shown in our analysis is also well established. It is another property of pulse rather than bursts itself. Whereas Ryde (2005) found an inverse relation between flux and lag. It is interesting that the three quantities correlate with each other. If pulse width and spectral lag can be viewed as mutual surrogates indeed the anti-correlated relation between $w$ and photon flux must be established. Similar result given by Hakkila et al. (2008) indicated that there has a correlation between the pulse duration $w$ and isotropic pulse peak luminosity. These pulse properties may give a useful constrain on theoretical model. Therefore, the correlation flux versus $w$ shown in Figure 7 is established in our sample.

The correlation flux vs. $w$ shown in Figure 7 seems neat, and we might suspect that it is just the result of a selection bias since other pulses, such as short-dim pulses are likely under-represented? Paper I examined the fluence hardness ratios integrated over the whole burst, with the split at $\tau_{\text {lag }}<1 \mathrm{~s}$ in the range $0.5<F_{\text {peak }}<2.0$ to see if there is any difference between long-lag and short-lag dim bursts. They found that the dim short-lag bursts have slightly harder spectra than the dim long-lag bursts in the same peak flux range. The analysis of Shahmoradi \& Nemiroff (2010) showed that simple hardness ratios are good estimator for the spectral peak energy in GRBs and it is independent of the type of the burst, whether long-duration GRB or short-duration. So the short-lag dim bursts should be greater peak energy than that of long-lag dim bursts. Whereas the mean peak energies of the burst spectra are correlated with intensity (flux): lower intensity groups of burst spectra 
exhibit a lower average peak energy (Mallozzi et al. 1995). Hence it is suggested that the short-lag dim bursts with greater peak energy might have higher intensity. Although the above properties of correlation are bursts rather than pulses we still think the correlation should be exist among pulses since Borgonovo \& Björnsson (2006) showed that the overall properties of a burst is determined mainly by the properties of pulses. Therefore, we tend to believe it is not a selection bias even if we can not give a test with a short-dim pulse sample.

The evident anti-correlation between the evolutionary slope during the pulse decay phase and pulse asymmetry seems to show pulses with short rise and very long decay times tend to more slower decay of $E_{p}$. The tendency appears to suggest the pulses might represent external shocks capable of initiating afterglow (Hakkila et al. 2008).

\section{Acknowledgments}

We thank the anonymous referee for constructive suggestions. This work was supported by the Science Fund of the Education Department of Yunnan Province (08Y0129), the National Natural Science Foundation of China (No. 10778726), the Natural Science Fund of Yunnan Province (2009ZC060M). 

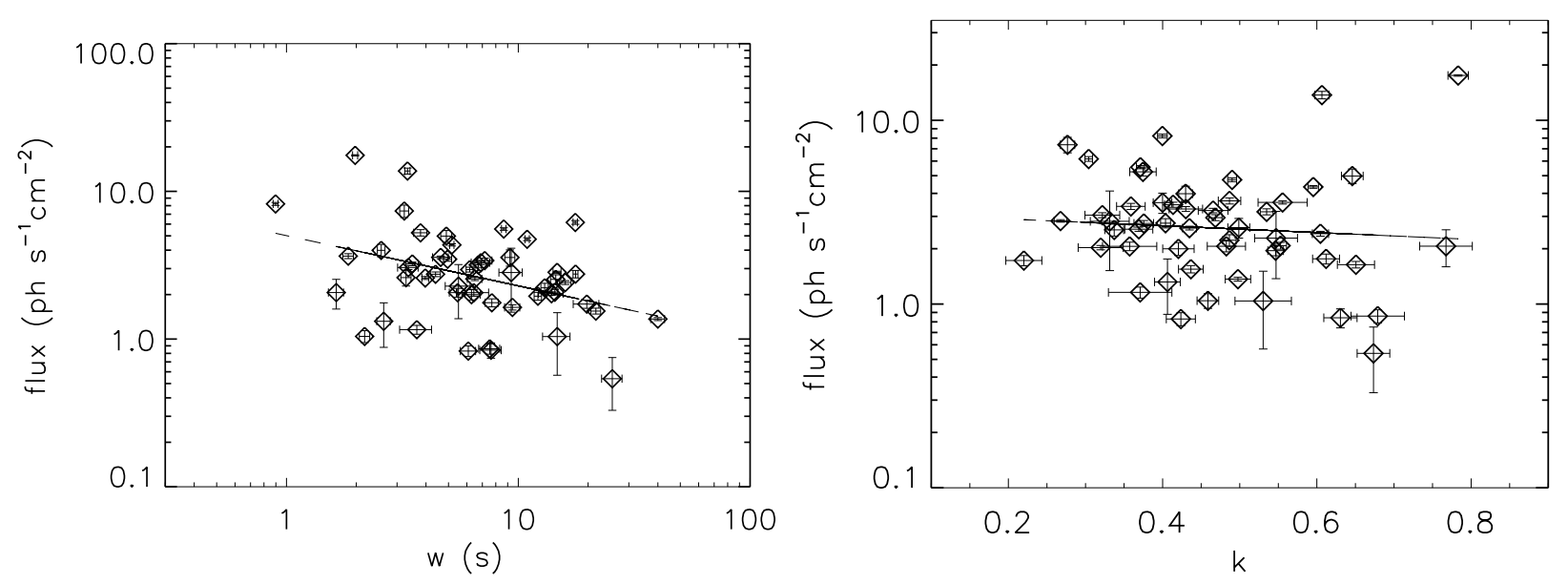

Fig. 7.- The photon flux vs. pulse width, $w$, (top panel) as well as photon flux vs. pulse asymmetry, $k$, for our sample, where the long dashed lines represent the best fitting lines. 


\section{REFERENCES}

Band, D., et al. 1993, ApJ, 413, 281

Borgonovo, L., \& Ryde, F., 2001, ApJ, 548, 770

Borgonovo, L \& Björnsson, C. I., 2006, ApJ, 652, 1423

Golenetskii, S. V., Mazets, E. P., Aptekar, R. L., Ilinskii, V. N., 1983, Nature, 306, 451.

Hakkila, J., Giblin, T. W., Norris, J. P., Fragile, P. C., Bonnell, J. T. 2008, ApJ, 677, L81

Kocevski, D., Ryde, F., \& Liang, E. 2003, ApJ, 596, 389

Kouveliotou, C., et al., 1993, ApJ, 413, L101

Mallozzi, R. S., et al. 1995, ApJ, 454, 597

Norris, J. P., et al. 1996, ApJ, 459, 393

Norris J. P., Bonnell J. T., \& Watanabe K., 1999, ApJ, 518, 901

Norris, J. P., et al. 2005, ApJ, 627, 324 (Paper I)

Peng, Z. Y., et al. 2006, MNRAS, 368, 1351

Peng, Z. Y., et al. 2007, ChjAA, 7, 428

Peng, Z. Y., et al. 2009a, ApJ, 698, 417 (Paper II)

Peng, Z. Y., et al. 2009b, NewA, 14, 311

Ryde, F., et al. 2005, A\&A, 432, 105

Ryde, F., 2005, A\&A, 429, 869

Quilligan, F., 2002, A\&A, 385, 377 
Shahmoradi, A., \& Nemiroff, R. J., 2010, arXiv:1002.0480v2 [astro-ph.HE]

Stern, B., Poutanen, J., \& Svensson, R., 1999, ApJ, 510, 312 